\title{
What are Implicit Definitions?
}

\section{Eduardo N. Giovannini ${ }^{1}$ (i) . Georg Schiemer ${ }^{2}$}

Received: 26 December 2018 / Accepted: 16 September 2019 / Published online: 8 November 2019

(c) The Author(s) 2019

\begin{abstract}
The paper surveys different notions of implicit definition. In particular, we offer an examination of a kind of definition commonly used in formal axiomatics, which in general terms is understood as providing a definition of the primitive terminology of an axiomatic theory. We argue that such "structural definitions" can be semantically understood in two different ways, namely (1) as specifications of the meaning of the primitive terms of a theory and (2) as definitions of higher-order mathematical concepts or structures. We analyze these two conceptions of structural definition both in the history of modern axiomatics and in contemporary philosophical debates. Based on that, we give a systematic assessment of the underlying semantics of these two ways of understanding the definiens of such definitions, by considering alternative model-theoretic and inferential accounts of meaning.
\end{abstract}

Keywords Modern mathematics · Formal axiomatics · Implicit definitions · Structural definitions $\cdot$ Model-theoretic semantics $\cdot$ Inferential semantics

Authors are listed in alphabetical order; the paper is fully collaborative.

Georg Schiemer

georg.schiemer@univie.ac.at

Eduardo N. Giovannini

engiovannini@conicet.gov.ar

1 CONICET and Universidad Nacional del Litoral, Bv. Pellegrini 2750, 3000 Santa Fe, Argentina

2 Department of Philosophy, University of Vienna, Universitätsstrasse 7, 1010 Vienna, Austria 


\section{Introduction}

The notion of implicit definition is a fundamental concept in modern mathematics, in particular in formal axiomatics. ${ }^{1}$ It is also ubiquitous in contemporary philosophical debates, ranging from the fields of philosophy of science and mathematics to philosophy of logic and language. As examples of the importance of this notion one can mention the debate on the meaning of theoretical terms in science, the contributions by Michael Friedman (and others) on the relativized a priori, as well as recent work in the philosophy of mathematics. ${ }^{2}$ Concerning the latter field, implicit definitions play a central role in neologicism and the discussion of abstraction principles as well as in mathematical structuralism. Finally, the notion features prominently in epistemology, in particular, in Boghossian's work on epistemic analyticity (e.g. Boghossian 1997).

Despite the relevance of this notion, it is surprising that it is commonly used very informally in these philosophical debates. The aim of this paper is to make more precise what implicit definitions are. We will address this issue by distinguishing between several types of definitions which are usually associated with the label "implicit definition". In particular, the focus here will be on a kind of definition used in formal axiomatics, which in general terms is usually understood as providing a definition of the primitives terms of a given axiomatic theory. In the context of the paper, we will label this sort of definition "structural definitions".

A central claim of this paper is that it is unclear how such definitions should be understood semantically. More precisely, we propose that both in modern mathematics and in subsequent philosophical debates one can distinguish between two ways of understanding structural definitions, namely (1) as specifications of the meaning of the primitive terms of a theory and (2) as definitions of higher-order concepts of mathematical objects, e.g. the concepts of a natural or real number system. The central aim in the following will be to survey these two conceptions of structural definitions both in the history of mathematics and in selected philosophical debates.

The paper is organized as follows. Section 2 presents a brief exposition of the logic of implicit and structural definitions. In Sect. 3, we then turn to a more detailed survey of the two conceptions of structural definitions, namely as definitions of the meaning of primitive terms of a theory (Sect. 3.1) and as definitions of higher-order concepts (Sect. 3.2). Section 4 contains a more systematic assessment of these two ways of thinking about structural definitions. In particular, in Sect. 4.1, we propose

\footnotetext{
${ }^{1}$ In this paper we use the expression "formal axiomatics" to refer more generally to the modern or abstract conception of the axiomatic method, inaugurated by Hilbert's investigations on the foundations of geometry (Hilbert 1899) and the arithmetic of real numbers (Hilbert 1900a). In these early developments, formal axiomatics was characterized by a formal understanding of mathematical language and the search for rigorous but non-formal proofs. In its mature mathematical form, formal axiomatics requires the use of a formal language, an explicitly formulated logical calculus, as well as a formal semantics. For the early developments of "formal axiomatics" see, e.g., Awodey and Reck (2002) and Sieg and Schlimm (2014).

${ }^{2}$ Compare, e.g., Friedman (1999). See Shapiro (1997) for a discussion of the role of implicit definitions in mathematical structuralism.
} 
two model-theoretic explications that capture the informal accounts presented in the previous section. Section 4.2 outlines an alternative, inferentialist account of the semantics of structural definitions. Section 5 presents some concluding remarks.

\section{What Axiom Systems Define}

A definition is generally understood as a statement or a set of statements that determine the meaning of an expression. Our focus here will be on stipulative definitions as used in mathematics and sometimes also in philosophical contexts. Such definitions are usually understood to establish the semantic interpretation of non-logical constants in a formal language. Expressions defined in this sense can be either relational or functional symbols (of a given arity) or individual constants of the language in question.

In modern logic, one usually draws a distinction between two types of such definitions, namely explicit and implicit definitions. Explicit definitions are statements of a specified syntactical form that have to meet the two standard criteria of adequacy for definitions, namely non-creativity and eliminability. ${ }^{3}$ Implicit (or contextual) definitions, in turn, are usually understood as sets of sentences that specify the interpretation of a newly introduced term contextually or holistically, that is, as a function of the meaning of the defining statements. ${ }^{4}$ Unlike explicit definitions, they do not need to satisfy any syntactic constraints.

In this paper, our focus will be on a specific kind of stipulative definitions related to axiomatic theories. For this purpose, it makes sense to draw a finer distinction between two types of implicit definitions that are often conflated with each other in the literature (cf. Gabriel 1978). We will label the first type implicit definitions in the strict sense and the second type structural definitions. ${ }^{5}$ Historically speaking, both methods were first developed in the context of modern formal axiomatics in the nineteenth-century.

Structural definitions concern the axiomatic specification of the primitive nonlogical terms of a given mathematical theory. Starting with the work of Hilbert and others, it is usually held that a formal axiom system provides a holistic definition of all of the primitive symbols used in the formulation of the theory. Thus, the axioms of Peano arithmetic serve as a definition of the primitive arithmetical terms 'natural number', 'zero', and 'successor'; Hilbert's axiom system of Euclidean geometry serves as a definition of the primitive geometrical terms 'point', 'line', 'plane', as well as of the relation symbols 'incidence', 'betweenness', 'congruence', etc.; ZFC defines the membership predicate ' $\in$ ' in the language of set theory, and so on.

\footnotetext{
3 Compare, e.g., Antonelli (1998) and Belnap (1993).

4 Compare Antonelli on this point: "[A]ny sentence containing a new symbol could be taken to be a candidate for a definition of that symbol. Such definitions are called 'contextual', in that the meaning of the new symbol is specified (at least partially) by giving the meaning of some larger expression in which it occurs; the symbol itself, when occurring in isolation, need not be endowed with any meaning at all" (Antonelli 1998, p. 151).

5 See Shapiro (unpublished) for a similar distinction.
} 
Implicit definitions in the strict sense, in contrast, are sentences (or collection of sentences) through which the interpretation of a term is specified in terms of one or several already interpreted terms. Thus, such definitions presuppose that all terms except the defined one are already interpreted. Implicit definitions in this sense were first introduced in the work of Gergonne and later by Padoa and are also often specified in axiomatic form. ${ }^{6}$ Roughly speaking, a set of axioms is taken to implicitly define a term if the axioms specify a unique interpretation of the term (relative to the given interpretation of the primitive vocabulary in the language in question).

To get a better idea of the difference between implicit definitions in the strict sense and structural definitions, it will be useful to present both notions more formally and to compare them with the standard specification of explicit definitions. ${ }^{7}$ Let $\mathcal{L}$ be a (not necessarily first-order) language with signature $\left\{\beta_{1}, \ldots, \beta_{m}\right\}$ and let $R$ be a relation symbol (of arity $n$ ) not contained in $\mathcal{L}$. Let $\mathcal{L}^{*}=\mathcal{L} \cup\{R\}$ be the expanded language containing $R$. Moreover, let $T$ be a theory (understood here in the usual sense as the closure of a given set of sentences under semantic consequence) expressed in $\mathcal{L}^{*}$.

Definition 1 (Explicit definition) The relation symbol $R$ is explicitly defined by $T$ in terms of $\mathcal{L}$ if a sentence in $\mathcal{L}^{*}$ of the form:

$$
\forall x_{1}, \ldots, x_{n}\left(R\left(x_{1}, \ldots, x_{n}\right) \leftrightarrow \Phi\left(x_{1}, \ldots, x_{n}\right)\right)
$$

is a consequence of $T$ (with $\Phi$ is a sentence in $\mathcal{L}$ ).

An implicit definition in the strict sense of $R$ in terms of the base language $\mathcal{L}$ also consists of sentence or a set of sentences of the expanded language $\mathcal{L}^{*}$. As mentioned above, the sentences in $T$ implicitly defining $R$ do not need to have a particular grammatical form as in the case of explicit definitions. Instead, implicit definitions are usually defined model-theoretically, that is, in terms of a constraint on possible interpretations of $T$. More formally, we say that:

Definition 2 (Implicit definition) The relation symbol $R$ is implicitly defined by $T$ in terms of $\mathcal{L}$ if for any two $\mathcal{L}^{*}$-structures $M, N$ that are models of $T$, we have:

if $M|\mathcal{L}=N| \mathcal{L}$ then $R^{M}=R^{N}$.

\footnotetext{
${ }^{6}$ Padoa (1900) might be credited as presenting the first sharp conceptualization of the notion of implicit definition in a strict sense. Moreover, he also provided a method for proving the indefinability of a primitive term of a formal theory from the remaining primitives of that theory, which later had a significant reception in Tarski's theory of definitions. For details, see Hodges (2008). See Gabriel (1978) for a historical discussion of the relation between implicit definitions in the tradition of Gergonne and structural definitions or "definitions through axioms".

7 In the following, we strongly draw on the definitions presented in Hodges (1993). For simplicity, we restrict our attention here to the definition of relation symbols. Our discussion can easily be generalized to hold also for other types of non-logical constants.

${ }^{8} M \mid \mathcal{L}$ and $N \mid \mathcal{L}$ present the $\mathcal{L}$-reducts of the $\mathcal{L}^{*}$-structures $M$ and $N$. See again Hodges (1993), p. 149.
} 
The interpretation of predicate $R$ is specified here in terms of the base language in the following way: a theory implicitly defines the predicate in case that any two models that assign the same interpretation of each of the terms $\left\{\beta_{1}, \ldots, \beta_{m}\right\}$, also agree on the interpretation of $R$. Put differently, a theory implicitly defines $R$ if it uniquely determines its interpretation relative to a given interpretation of the nonlogical symbols of the base language. ${ }^{9}$

Turning to structural definitions, we mentioned that such definitions are usually understood as simultaneous specifications of the primitive non-logical vocabulary of a given language in terms of an axiomatic theory. More specifically, given our choice of terminology, we can say that the primitive terms $\beta_{1}, \ldots, \beta_{n}$ of some theory $T^{\prime}$ expressed in the base language $\mathcal{L}$ are structurally defined by the axioms of $T^{\prime}$. A central requirement for the adequacy of such structural definitions is that $T^{\prime}$ itself is consistent (in the semantic sense of the term), that is, that there exists an interpretation of $\mathcal{L}$ that forms a model of $T^{\prime}$.

Let us consider a mathematical example from real analysis to illustrate these two types of implicit definitions. Ordered fields are structures of the form $\langle R, 0,1,+, \cdot, \leq\rangle$ consisting of a set $R$, an operation of addition + , an operation of multiplication ', and an order relation $\leq$, such that addition and multiplication are associative and commutative, 0 is the identity of addition, 1 the identity of multiplication, multiplication is distributive over addition, every element has an additive inverse, every element different from 0 has a multiplicative inverse, $\leq$ is a total order, and the elements greater than or equal to 0 are closed under addition and multiplication. A (first-order) axiomatization of ordered fields can be specified as follows: ${ }^{10}$

Example 1 (Ordered fields) Let $\mathcal{L}$ be the language of ordered fields with signature $\{0,1,+, \cdot, \leq\}$. The theory of ordered fields is axiomatized as follows:
(F1) $\forall x \forall y \forall z((x+y)+z=x+(y+z))$
(F2) $\forall x \forall y \forall z((x \cdot y) \cdot z=x \cdot(y \cdot z))$
(F3) $\forall x \forall y(x+y=y+x)$
(F4) $\forall x \forall y(x \cdot y=y \cdot x)$
(F5) $\forall x(x+0=x)$
(F6) $\forall x(x \cdot 1=x)$
(F7) $\forall x \exists y(x+y=0)$
(F8) $\forall x(x \neq 0 \rightarrow \exists y(x \cdot y=1))$
(F9) $\forall x \forall y \forall z(x \cdot(y+z)=(x \cdot y)+(x \cdot z))$
(F10) $\forall x \forall y \forall z((x \leq y) \wedge y \leq z) \rightarrow x \leq z)$
(F11) $\forall x \forall y(x \leq y \vee y \leq x)$
(F12) $\forall x \forall y((x \leq y \wedge y \leq x) \rightarrow x=y)$
(F13) $\forall x \forall y((0 \leq x \wedge 0 \leq y) \rightarrow(0 \leq x+y \wedge 0 \leq x \cdot y))$

Moreover, if a (second-order) axiom of completeness is added to the (first-order) axioms of ordered fields, one obtains an axiomatization of a complete ordered field.

\footnotetext{
${ }_{9}^{9}$ In the context of first-order theories, Beth's definability theorem shows that explicit and implicit definitions are equivalent. It follows that implicit definitions in the strict sense also satisfy the criteria for explicit definitions, namely eliminability and non-creativity. See, e.g., Hodges (1993, pp. 301-302) for further details.

${ }^{10}$ This axiomatization has been provided by Shapiro (1991). For an alternative axiomatization, see Tarski (1994).
} 
A common alternative is to postulate, for instance, the following least upper bound axiom, which asserts that every bounded set has a least upper bound:

$$
\text { (F14) } \forall X([\exists y X(y) \wedge \exists z \forall y(X(y) \rightarrow y \leq z)] \rightarrow \exists z \forall y(\exists u(X(u) \wedge y \leq u) \vee z \leq y))
$$

Now, it should be noted that, in the above axiom system for complete ordered fields, the elements of the signature $\{0,1,+, \cdot, \leq\}$ are not all independent, but three of them can be defined in terms of the others. More specifically, the order relation ' $\leq$ ' can be explicitly defined in terms of addition and multiplication as follows:

$$
\forall x \forall y[x \leq y \leftrightarrow \exists z(x+(z \cdot z)=y)]
$$

Then, theory (F1-F14) gives an implicit definition (in the strict sense) of the term ' $\leq$ ' with respect to $\mathcal{L} /\{\leq\}$. A central aspect here is that in the theory of complete ordered fields the ordering relation is uniquely determined. Analogously, explicit definitions of the individual constant ' 0 ' and ' 1 ' can also be given in terms of multiplication and addition. ${ }^{11}$ Moreover, since these axioms specify the notion of a complete ordered field, the theory $(\mathrm{F} 1-\mathrm{F} 14)$ can be viewed as a structural definition of the terminology of complete ordered fields in $\mathcal{L}$ with signature $\{0,1,+, \cdot, \leq\}$.

This example illustrates nicely how the two types of axiomatic definitions differ from each other. Two points should be mentioned here. First, implicit definitions in the strict sense and structural definitions can be viewed as two ways in which an axiom system can act as a definition of non-logical terms. In the case of implicit definitions, we saw that axioms provide a definition of a particular term in terms of the primitive terms of the theory. In the case of structural definitions, no semantic specification of a base language is presupposed. Here, an axiom system is taken to holistically specify the meaning of the primitives independently of any prior semantic stipulations.

The second point also concerns an important difference in the semantic understanding of structural and implicit definitions. The latter type of definition is usually specified model-theoretically by fixing the reference of the newly introduced term. More specifically, a theory is said to implicitly define a term relative to a given signature just in case it uniquely determines the reference of the term relative to models with the same interpretation of the primitive terms. Thus, the model-theoretic condition expresses a requirement of the uniqueness of reference for implicitly defined terms.

In contrast, in the case of structural definitions, this kind of uniqueness of reference is deliberately ruled out. Starting with Dedekind's and Hilbert's work, there is a consensus in the literature on formal axiomatics that axiom systems-taken as structural definitions of primitive terms-do not have to fix a unique reference of these terms. Rather, their specification through an axiom system is deliberately left

\footnotetext{
${ }^{11}$ The symbols ' 0 ' and ' 1 ' can be explicitly defined in terms of addition and multiplication as follows:

1. $x=0 \leftrightarrow \forall y(y+x=y)$

2. $x=1 \leftrightarrow \forall y(y \cdot x=y)$

For details see Tarski (1994).
} 
semantically indeterminate. Compare, for instance, Hilbert's famous remark on this issue in a letter to Frege of 1899:

You say my concepts, e.g., 'point', 'between', are not unambiguously determined; (...) Yes, it is obvious that any theory is actually only a framework or a schema of concepts, together with the necessary relations of these concepts to each other, and the base elements can be thought of in an arbitrary way. If, as my points, I think of some system of things, e.g., the system: love, law, chimney sweep... and then assume my axioms as relations between these things, then my theorems, too, hold of these things, e.g., the Pythagorean Theorem. In other words, any theory can always be applied to infinitely many systems of basic elements. (Hilbert to Frege, 29 December 1899, reprinted in: Frege (1980))

The indeterminacy of reference embraced by Hilbert clearly distinguishes structural definitions from implicit definitions in the strict sense. At the same time, it leaves us puzzled about the proper semantic understanding of the former kind. ${ }^{12}$ Whereas the referential semantics of implicitly defined terms is well understood, it is not clear, at least semantically, how structural definitions work. Put differently, given that such definitions do not fix the reference of the defined terms, what do structural definitions actually define?

\section{Two Notions of Structural Definitions}

The aim of this section is to distinguish between two ways of understanding the semantics of structural definitions. The first way conceives structural definitions as determining the meaning of the primitive terms of a mathematical theory. The second way considers structural definitions as definitions of higher-order concepts of mathematical objects, or more precisely, of a class of models for a given theory. We will show that these two ways not only can be found in historical formative work in formal axiomatics, but that they are also widespread in the contemporary philosophical literature.

\subsection{Structural Definitions of Primitive Terms}

A first way of understanding the semantics of structural definitions consists in claiming that the axioms of a mathematical theory define or establish the meaning of the primitive terms. Historically, this conception of structural definitions is related to methodological innovations triggered by the emergence of the modern axiomatic method. This view is also present in contemporary philosophical literature, but

12 On the thesis of the indeterminacy of reference in modern axiomatics see Hallett (2010). 
usually explained or illustrated in an informal fashion. In this section we do not aim at presenting a detailed historical analysis of the occurrence of this understanding of structural definitions in key historical figures, but just to illustrate our contention with some representative textual support. ${ }^{13}$ As we will see, while several authors refer to the "meaning of primitive terms" when discussing axiomatic definitions, they usually leave unspecified what constitutes the meaning of a mathematical term. We will return to this issue in Sect. 4.1.

One of the most important and original methodological innovations which are commonly related to David Hilbert's early works in formal axiomatics, especially in his influential Foundations of Geometry (1899), is the introduction and systematic use of the so-called "method of implicit definitions". ${ }^{14}$ In the explanatory remarks of that booklet, Hilbert asserts that "the axioms of this group [i.e., axioms of order] define the concept of 'between"'(Hilbert 1971, p. 5) and that "the axioms of this group [i.e., axioms of congruence] define the concept of congruence and with it that of displacement" (Hilbert 1971, p. 10). Moreover, the key idea of this new conception of mathematical definitions is concisely expressed in the first lines of the classic monograph:

Consider three distinct sets of objects. Let the objects of the first set be called points... the objects of the second set be called lines... and the objects of the third set be called planes.

[...] The points, lines and planes are considered to have certain mutual relations and these relations are denoted by words like "lie", "between", "congruent", The precise and mathematically complete description of these relations follows from the axioms of geometry. (Hilbert 1971, p. 3)

And a similar description can also be found in the Paris conference on "Mathematical Problems" of 1900:

When we are engaged in investigating the foundations of a science, we must set up a system of axioms which contains an exact and complete description of the relations subsisting between the elementary concepts [elementare Begriffe] of that science. The axioms so set up are at the same time the definitions of those elementary concepts. (Hilbert 1900b, p. 447)

Hilbert's assertion that the axioms of a mathematical theory should be taken as the definition of its primitive concepts is a consequence of his novel view of the schematic nature of mathematical primitives. According to the abstract

\footnotetext{
13 For a general outline of the emergence of the notion of structural definitions in modern axiomatics see Menahem (2006).

14 It is well known that Hilbert did not use the expression "implicit definition" in published work. However, we located at least one use of this expression in the notes for the lecture course "Wissen und mathematisches Denken", held in the winter semester of 1922/1923: "Already when we consider the position of three points [on a line], we recognize the fact that "betweenness" is evidently the fundamental concept, the deciding criterion. For this purpose, we lay down the following axioms, which implicitly define this [concept]" (Hilbert 1988, p. 82. Our emphasis). For the introduction of the term "implicit definition" see Gabriel (1978).
} 
conception of the axiomatic method, mathematical primitive concepts and relations do not have a pre-theoretical or pre-axiomatic given sense; on the contrary, all mathematically relevant properties are established in the axioms. Neither have the primitive terms a given fixed reference or extension, but they are free to receive multiple and diverse interpretations, as long as these interpretations make the axioms true. However, the schematic character of the mathematical primitives does not imply for Hilbert that they should be regarded as mere symbols without meaning. Geometrical axioms bestow a meaning to geometrical primitives such as 'line', 'point', 'plane', etc., since they determine or fix the range of their possible interpretations. This idea is usually illustrated in an informal fashion by claiming that, in Hilbert's axiomatization of geometry, the geometrical primitive terms designate whatever entities satisfy the relations established in the axioms. In other words, the axioms serve as the definition of the mathematical primitives, insofar as they stipulate the meaning they must have in order to make the axioms true.

In response to a series of critiques raised by Frege in a well-known letter exchange, Hilbert attempted to clarify his viewpoint concerning axiomatic definitions. Some brief comments on this are in order. First, an important aspect of Hilbert's conception of structural definitions is that the fundamental properties or characteristics of each particular primitive concept are established by means of its relations to the other primitives of the theory, as they are described in the axioms. This implies that the axioms stipulate the meaning of the primitives holistically-so to say, since the "meaning" of every concept consists in its relations to the other primitives. Hilbert expresses this idea, in one of his replies to Frege, as follows:

In my opinion, a concept can be fixed logically only by its relations to other concepts. These relations, formulated in certain statements, I call axioms, thus arriving at the view that axioms (perhaps together with propositions assigning names to concepts) are the definitions of the concepts. (Hilbert to Frege, 22 September 1900, reprinted in: Frege (1980))

Second, Hilbert makes plain that the whole axiomatic system, and not a particular axiom or group of axioms, must be regarded as the definition of the primitive concepts and relations. A complete understanding of the primitives requires the totality of the axioms, since each particular axioms brings something to the "content" of the primitives. This view is essential to Hilbert's systematic method of proving independence results by means of the construction of different numerical "models" or interpretations of the geometrical axioms. As Hilbert explains to Frege,

Every axiom contributes something to the definition, and hence every new axiom changes the concept. A 'point' in Euclidean, non-Euclidean, Archimedean and non-Archimedean geometry is something different in each case. (Hilbert to Frege, 29 December 1899, reprinted in: Frege (1980)) 
Finally, Hilbert establishes explicitly that consistency is a necessary and sufficient condition of adequacy and success of structural definitions. As long as the properties of the primitives established in the axioms do not contradict each other, and thereby the axiomatic system proves to be consistent, the axioms accomplish the goal of defining the primitive concepts and relations occurring in them. However, it should be noted that, at this early stage, Hilbert did not have a sharp distinction between syntax and semantics. Accordingly, it is not clear whether he was conceiving 'consistency' in a more syntactic sense, as deductive consistency, or in a semantic sense, as satisfiability. ${ }^{15}$ In any case, an essential aspect of this initial model of structural definitions is that the consistency of the axioms is not a matter of stipulation. On the contrary, according to Hilbert, only in the case one has proved that the axioms are consistent (e.g., satisfiable), one can legitimately claim that they provide a successful structural definition of the primitive concepts and relations.

The conception of structural definitions as definitions of the primitive terms of a mathematical theory was even more explicitly formulated by the members of the Peano School, by the end of the nineteenth century and the beginning of the twentieth century. For instance, in a talk pronounced at the International Congress of Philosophy in Paris, in 1900, Burali Forti addressed the problem of definitions in mathematics. He distinguished there three sorts of definitions: "nominal definitions", "definitions by postulates" and "definitions by abstraction". Regarding the second kind of definitions, Burali Forti offered the following characterization:

One uses the definition by postulates for a grouping $x$ of objects, when we do not know or do not want to define them nominally. The group $x$ is defined by postulates by means of the logical relations among the $x$. M. PEANO defined, in this way, the group of words point, segment, and the group of words whole number, zero, successor of a number, and M. PIERI the group of words point, movement. (Burali 1901, p. 295.)

According to this view, the meaning of mathematical primitives in a (formal) axiomatic theory is then constituted by their mutual logical relations, as they are established in the axioms. Moreover, this notion of definition by postulates or axioms was soon after taken up by F. Enriques, who was the first to link this conception of structural definitions with the term "implicit definition". 16

\footnotetext{
${ }^{15}$ Hilbert's semantic interpretation of consistency is suggested, for example, by the following famous claim in his controversy with Frege: "if the arbitrary given axioms do not contradict one another with all their consequences, then they are true and the things defined by the axioms exists" (Hilbert to Frege, 29 December 1899, reprinted in: Frege 1980). A more syntactic reading of Hilbert's early view of consistency has been defended by Sieg (2009). On Hilbert's early metatheoretical view of axiomatic systems see, e.g., Awodey and Reck (2002) and Blanchette (2017).

${ }^{16}$ In an influential article for the Encyklopädie der mathematischen Wissenschaften (vol. III, 1.1), published in 1907, Enriques claimed: "This conception of rigor [...] is exhibited in the abstract logical viewpoint according to which the postulates appear as arbitrary agreements, and the totality of logical relations which they express, constitutes a sort of implicit definition of the primitive concepts" (Enriques 1907, p.11).

According to Gabriel (1978), Enriques is responsible not only for the introduction of the expression "implicit definition" in the German speaking world, but also for the confusion between implicit definition (in the strict sense) and structural definitions in the first sense, that is, as definitions of primitive mathematical concepts.
} 
Hilbert's new method of "implicit definitions" exerted a remarkable influence among early twentieth-century philosophers of science and mathematics. In particular, Moritz Schlick argued that this new kind of definitions in mathematics was a fundamental tool to ground our mathematical knowledge on a secure and a priori basis. In his General Theory of Knowledge (1974), Schlick makes the following remark:

Hilbert's geometry begins with a system of propositions in which a number of terms occur (such as 'point', 'straight-line', 'plane', 'between', 'outside of', and the like) that, to begin with, have no meaning or content. These terms acquire meaning only by virtue of the axiom system, and possess only the content that it bestows upon them. They stand for entities whose whole being is to be bearers of the relation laid down by the system. (Schlick 1974, p. 34)

For Schlick, the method of "implicit definitions" was a fundamental methodological tool to avoid the appeal to intuition and experience in the formation and determination of mathematical and scientific concepts, thereby contributing to the acquisition of rigorous and certain knowledge. ${ }^{17}$ This appeal was avoided due to the fact that, by means of this definitional method, the basic or simple mathematical concepts were deprived of their original intuitive meaning, namely of their ordinary sense as well as their usual denotation. On the contrary, by considering that an (abstract) system of axioms provided an implicit definition of its primitive concepts, the meaning or "content" of each primitive concept consisted now exclusively in its logical relations to the other concepts as expressed in the axioms. According to Schlick, this provided a complete and exact determination of all simple mathematical concepts, although they were not endowed anymore with a fixed or intuitive reference.

It is worth noting that, although the above distinguished promoters of this initial model of structural definition agreed that, when successful, this new kind of definition provided a meaning to the primitive terms of a mathematical theory, the very notion of 'meaning' invoked here was rarely made clear or even explicitly analyzed within these early discussions. ${ }^{18}$ On the contrary, the common standpoint during the period was limited to the suggestion that, since structural definitions do not fix the reference of the defined terms, their meaning or "content" consisted now in the mutual (logical) relations that each primitive concept bore to the other primitive concepts. In the following Sect. 4.1, we will focus more closely on this "holistic" nature of the meaning involved in this first conception of structural definitions.

The conception of structural definitions as definitions of the meaning of primitive terms is very much alive and widespread in the contemporary philosophical literature. This contention can be illustrated by indicating two interesting receptions and reinterpretations of this view of "implicit definitions", stemming from epistemology

\footnotetext{
17 For the significance of the doctrine of "implicit definitions" in the development of logical empiricism, and particularly for Schlick, see Coffa (1991).

18 Of course, the main exception to this claim was Frege, who on the basis of his fully elaborated semantical theory rejected this first understanding of structural definitions. See Frege (1980).
} 
and philosophy of mathematics and logic. The first case is related to the current philosophical discussion of the notion of analyticity and a priori knowledge. In particular, Boghossian $(1996,1997)$ has put forward a concept of analyticity on the basis of an implicit definition account of the meaning of logical constants. According to this epistemological concept of analyticity, a statement $S$ is analytic if the mere grasp of the meaning of $S$ is a sufficient condition for justified belief in its truth. ${ }^{19}$ Then, Boghossian has argued that an analytic theory of our a priori knowledge of logical truths can be sustained, by appealing to the doctrine that logical axioms-or certain rules of inference-constitute an implicit definition of the logical constants occurring in them. The central notion of implicit definition is specified as follows:

Implicit definition: it is by arbitrarily stipulating that certain sentences of logic are to be true, or that certain inferences are to be valid, that we attach a meaning to the logical constants. More specifically, a particular constant means that logical object, if any, which makes valid a specified set of sentences and/or inferences involving it. (Boghossian 1997, p. 348)

The central contention here is that the meaning of logical constants is fixed or determined by stipulating that certain sentences or rules which contain them are respectively true or valid. If the implicit definition is successful, the logical constants acquire the meaning required for the sentences or logical rules to be respectively valid or true. Then, a novel ingredient of this model is the explicit stipulation of the deductive validity of the rules working as implicit definitions of the logical constants. It is worth mentioning that Boghossian takes this conception of implicit definition of logical constants as the immediate application, in the case of logic, of the method of determining the meaning of geometrical primitives or "indefinables" by means of the postulation of a set of axioms, as introduced in modern axiomatic geometry. Moreover, Gentzen's suggestion that the meaning of logical constants might be determined by the stipulation of the rules for their introduction and elimination is also commonly mentioned as an important motivation of the "standard" conception of implicit definition. ${ }^{20}$

This particular understanding of the notion of implicit definition is usually indicated in contemporary philosophical literature as the "standard conception" or the "standard model" of implicit definitions. ${ }^{21}$ The key idea of the standard model is that the decision to regard a certain sentence, or a set of sentences, as true is essential to provide the implicitly defined term with a meaning. In other words, the stipulative truth of the sentence, or set of sentences, determines the meaning that the implicitly defined term acquires, since in a successful implicit definition the definiendum gets the unique meaning required for the definiens to express truth. ${ }^{22}$

\footnotetext{
${ }^{19}$ Cf. Boghossian (1997, p. 334).

${ }^{20}$ For details of Gentzen's remark see below, Sect. 4.2.

21 See Horwich $(1997,1998)$.

22 In the literature it is often argued that this kind of implicit definition has a fundamental epistemological import, for it can be used to provide a satisfactory account of the possibility of a priori knowledge, particularly in logic and mathematics. The view that implicit definitions can underwrite important kinds of non-inferential a priori knowledge has been described as the "traditional connection". For details see, e.g., Hale and Wright (2000) and Ebert (2016).
} 
The second case of deployment of the concept of "implicit definition" in contemporary philosophical literature is connected with the neologicist program in the philosophy of arithmetic. For some neologicists - particularly Bob Hale and Crispin Wright - a special notion of implicit definition is fundamental to provide an account of our a priori knowledge in arithmetic. This is related to the fact that they consider the abstraction principle known as "Hume's principle" (henceforth HP) as an implicit definition of the notion of cardinal natural number. Informally, Hume principle asserts that two concepts have the same number if and only if the objects that fall under them can be put into a one to one correspondence. Formally, HP is usually formulated as follows:

$$
\forall F \forall G(\#(F)=\#(G) \Leftrightarrow(F \approx G)),
$$

where $F, G$ stand for concepts (or properties, or sets), i.e., they are second order variables; the symbol " $\approx$ " denotes the relation of numerical equivalence, or more precisely, the equivalence relation of equinumerosity of concepts, and the numeral symbol "\#" stands for a function that takes concepts as arguments and returns an object (a number) as a value. The expression "\#F" refers to a singular term which must be read as the number of $F$. Hence, HP stipulates an equivalence between the notion of numerical identity and the notion of biunivocal relation.

The main tenet defended by some neologicists, such as Hale and Wright, is that by means of the stipulation of the truth of HP, it is possible to grasp the meaning of the cardinal operator, which occurs two times in the left-hand side flanking an identity sign. As Hale and Wright observe: "by stipulating the truth of HP the sense or meaning of the numeral operator '\#' is implicitly defined or adequately explained" (Hale \& Wright 2001, p. 310. Our emphasis). It should be noticed that what is here stipulated is not simply the truth of HP. On the contrary, the model of meaningfixing stipulation involved in HP consists in asserting that the truth-conditions for numerical identity statements depend on the holding of a one-to-one correlation between a pair of concepts, i.e., on the equinumerosity relation between concepts. ${ }^{23}$

To conclude this section, it is worth asking how this general notion of "implicit definition" used in contemporary philosophical literature is related to the first conception of structural definitions, i.e., definitions of the meaning of the primitive terms of a given axiomatic theory. In particular, while in the former case the stipulation of the truth of a sentence, or a collection of sentences, usually fixes a meaning for a particular non-logical term there contained, in the latter case the laying down of an axiomatic system provides simultaneously a definition of the several nonlogical terms appearing in the axioms. In this regard, Ebert (2016) has pointed out recently that structural definitions-or "Hilbert-style" definitions-do not constitute a different sort of mathematical definition, but must be conceived just as a particular instance of the broader notion of implicit definition (or contextual definition). ${ }^{24}$ The

\footnotetext{
23 It should be noted that, although this conception of implicit definition presupposes a notion of validity, the latter notion is not specified in model-theoretic terms but rather taken as primitive. We thank an anonymous referee for calling our attention to this point. For a full discussion of the interpretation of HP as an implicit definition see Hale and Wright (2000), Ebert and Shapiro (2009) and MacFarlane (2009).

24 See Ebert (2016, pp. 137-138).
} 
alternative here is then to consider that a structural definition of the meaning of the primitive terms of an axiomatic theory is a collection of multiple implicit definitions (in a strict sense). We will investigate this issue more formally in Sect. 4.

\subsection{Structural Definitions of Higher-Order Concepts}

The second understanding of structural definitions holds that what an axiomatic system defines is not the meaning of its primitive terms, but a higher-order concept of mathematical objects. In modern terminology, a structural definition is a definition of the class of models of a given axiomatic theory. Historically, this conception also originated with the emergence of formal axiomatics, although it was not so explicitly formulated as the first view of structural definitions. In the contemporary scene, this understanding of structural definitions plays a central role in the structuralist conception in the philosophy of mathematics. In what follows we present some representative examples of this second view of the semantics of structural definitions, both from early formative work in modern axiomatics and its philosophical analysis.

Dedekind's seminal work on the foundations of arithmetic, in the classic essay Was sind und was sollen die Zallen? (1888), represents an early example of the conception of structural definitions as definitions of higher-order concepts of mathematical objects. This can be appreciated in the central definition [Erklärung] 771 of the concept of simply infinite system. For better readability, we state the definition using current terminology.

Definition 3 A set $S$ is said to be simply infinite if there exists a function $f$ on $S$ and an element $a \in S$ such that the following hold:

1. $f(S) \subseteq S$, i.e. $f$ is a mapping from $\mathrm{S}$ into itself.

2. $a \notin f(S)$, i.e., $a$ is not in the image of $S$ under $f$.

3. $f(x)=f(y)$ implies $x=y$, i.e., $f$ is a one-to-one function.

4. $S$ is the smallest set containing $a$ and closed under $f$, i.e., it is the intersection of all such sets. $^{25}$

Dedekind carefully avoided the use of the word 'axiom' to describe the requirements 1-4 in the above definition, and refers to them instead as "characteristic conditions" [characteristische Bedingungen]. However, it has been firmly established in the literature that his methodological approach clearly exhibits a modern understanding of the axiomatic standpoint. ${ }^{26}$ More specifically, Dedekind is very precise about the fact that this definition provides a general characterization of the

\footnotetext{
${ }^{25}$ We follow here the reconstruction provided by Awodey and Reck (2002, p. 6). It should be noted that the order of the conditions 1-4 do not obey Dedekind's original setting. Moreover, the condition 4 is formulated by Dedekind on the basis of his theory of chains, by stating that $S$ is the chain under $f$ with base point $a$. Cf. Dedekind $(1888, \S 71)$.

${ }^{26}$ For a detailed analysis of Dedekind's understanding of the modern axiomatic standpoint, see Sieg and Schlimm (2014) and Sieg and Morris (2018).
} 
higher-order abstract concept of simply infinite systems, and not a description of a particular system of objects which instantiates this concept. Moreover, in the following definition $\$ 73$, he defines the natural numbers as the "abstract type" of simply infinite systems. ${ }^{27}$ Dedekind can thus be considered a pioneer of the second understanding of structural definitions.

Another, at least partial, insight into this second understanding of structural definitions can be found in Frege's attempt to reconstruct the logical form of "Hilbert's definitions" in Grundlagen. As is well known, Frege plainly rejected the view that the axioms of geometry can provide a definition of the primitive geometrical terminology. Since in Hilbert's axiomatic theory the primitives do not have a fixed reference and are free for reinterpretation, its axioms do not express evident true propositions and cannot be considered as genuine geometrical axioms. Neither can Hilbert's axioms be regarded as definitions of the primitives, since they fail to deliver the main task of all definitions, i.e., to fix the reference and provide a sense for the defined expression. However, Frege suggested that an alternative view of the definitional character of axioms could be possible in principle. If Hilbert's axioms define anything at all, they define "second-level concepts" under which many different first-order concepts fall:

Now, how do things stand with Mr. Hilbert's definitions? Apparently every single point is an object. From this it follows that the concept of a point (is a point) is of the first level. If we now go through Mr. Hilbert's axioms, considering them as parts of the definition of a point, we find that the characteristics stated in them are not of the first level. That is, they are not properties an object must have in order to be a point. Rather, they are of the second level. Therefore, if any concept is defined by means of them, it can only be a secondlevel concept. It must of course be doubted whether any concept is defined at all, since not only the word 'point' but also the words 'straight-line' and 'plane' occur. (Frege 1903, p. 374. Our emphasis)

Frege understands "second-level concepts" as higher-level concepts under which other concepts, but no objects, fall. For instance, if an individual point is an object, then the concept of point is a first-level concept. However, Frege argues that the conditions formulated in Hilbert's axioms for the geometrical primitives do not aim to characterize first-level concepts, but second-level concepts, under which particular first-level concepts fall. More precisely, according to Frege, the first-order concepts "is a (Euclidean) point" and "is a pair of numbers from the domain $\Omega$ " could fall under a second-level concept, whose characteristic marks are established in Hilbert's axioms. ${ }^{28}$ Now, Frege's proposal can be generalized by noting that what is defined here is not a second-level concept, but a higher-order relation, which connects the geometrical primitives 'point', 'straight-line', 'plane', 'between', and so on. If this reading could be sustained, Frege's reconstruction of the logical form of Hilbert's

\footnotetext{
27 The specific nature of the process of abstraction involved in definition $\$ 73$ has been extensively discussed in the literature. For recent studies see Klev (2017) and Sieg and Morris (2018).

28 The domain $\Omega$ refers to Hilbert's construction of a minimal Pythagorean field. See Hilbert $(1899, \S 9)$.
} 
definitions would be very close to the modern view that axioms define (classes of) relational structures. ${ }^{29}$

Now, we have seen that Hilbert explicitly stressed the idea that his axioms establish the meaning of the primitive concepts and relations of the geometrical theory. Accordingly, it has often been emphasized in the literature that, although in practice Hilbert provides structural definitions of geometrical structures, he could not have foreseen this conception of structural definitions in this early period. ${ }^{30}$ However, a different picture emerges when Hilbert's unpublished notes for lecture courses on the foundations of mathematics, corresponding to this period, are taken into consideration. For example, the notes for the lecture course Logical principles of mathematical thinking (1905a, b), which contain the most complete description of his early view of the axiomatic method, are very informative in this regard. Concerning the axiomatic construction of the real number system, Hilbert makes the following remark:

In the construction of arithmetic we have deviated from its intuitive foundation, namely the concept of natural number, which constitutes the starting point of the genetic method. For us the number system was ultimately nothing but a scaffolding of concepts, which was defined by means of the 18 axioms. (Hilbert 1905a, p. 36. Our emphasis) $^{31}$

A similar observation can also be found in a second version of these lecture notes, in relation to the axiomatic system for Euclidean geometry:

We consider geometry as a system of things, which we designate as points, lines, etc. and we establish for them certain connections, by means of which the conceptual scaffolding of geometry is completely defined. (Hilbert 1905b, pp. 36-37. Our emphasis) ${ }^{32}$

These passages explicitly reveal that, in this early period, Hilbert also considered an alternative view of structural definitions as definitions of higher-order concepts of mathematical objects, i.e., classes of mathematical structures. The axiom system of Euclidean geometry provides a definition of a three dimensional Euclidean space, while his axioms for the real number system constitute a definition of a complete ordered field.

\footnotetext{
${ }^{29}$ This interpretation has recently been defended by Hallett (2012), who also offers a formal logical reconstruction of Frege's view. See also Eder (2016).

${ }^{30}$ Classical examples of this reading are Gabriel (1978) and Resnik (1974). Recently, Klev (2011) has also defended a similar view.

31 „Wir sind beim Aufbau der Arithmetik von deren anschaulicher Grundlage, dem Anzahlbegriff, schließlich ganz abgekommen, der ja noch bei der genetischen Methode den Ausgangspunkt bildet. Uns war das Zahlsystem schließlich nichts, als ein Fachwerk von Begriffen, das durch 18 Axiome definiert war".

32 „Man betrachtet die Geometrie als ein System von Dingen, die als Punkte, Gerade, etc. bezeichnet werden und legt für gewisse Verknüpfungen fest, durch deren Gesamtheit dann das begriffliche Fachwerk der Geometrie definiert ist“".
} 
Let us now turn again to the early philosophical reflection of modern axiomatics in the twentieth-century. The philosopher whose work contains the most detailed discussion of axiomatic definitions is Rudolf Carnap. More specifically, in Carnap's work on "general axiomatics" from the late 1920s, one finds a clear expression of the Fregean view that what it is defined by means of structural definitions is a higher-order concept or a class of models. For instance, this idea is expressed informally in his notes on a course titled Philosophy of Space; Foundations of Geometry, first presented in Vienna in 1928. Carnap describes the axiomatic method here as follows:

The AS [axiom system] is about undetermined objects. It determines only a relational structure between them.

Implicit definition: but more precisely: definition of a class of systems of objects, that is the shared "structure" of these systems. (RC 089-62-02)

Thus, an implicit definition in this sense does not specify the meaning of the primitive terms of a theory. Rather, it specifies the "structure" shared by all of its systems or models.

This distinction between structural definitions in the first sense and in the second sense is made more explicit in Carnap's published works from the time. In his "Eigentliche und uneigentliche Begriffe" (1927), Carnap refers to Schlick's discussion of axiomatic definitions mentioned above. Carnap argues here that an axiom system can be understood as giving an "implicit definition" of the primitive concepts of a theory. However, it is central for Carnap that the concepts defined in this way do not constitute "proper" but "improper concepts". They are improper in the sense that they remain indeterminate in an important sense: it is not possible to determine in isolation whether a particular object (or function) falls under one of the concepts in question. For instance, it is impossible to determine whether a particular function qualifies as an arithmetical successor function without taking into account the other concepts defined by the axiom system for arithmetic.

Whereas implicit definitions (understood here as structural definitions in our first sense) remain indeterminate, Carnap identifies a second sense in which an axiomatic theory can function as a definition that does not have this limitation. An axiom system, if consistent, not only implicitly defines its primitive terms, it also provides an explicit definition of a proper (and higher-order) concept. In the case of arithmetic, this is the concept of an arithmetical progression (i.e. in modern parlance, the concept of natural number system); in the case of Euclidean geometry, it is the concept of Euclidean space, etc.

The fact that each formal axiom system also defines a "proper concept" is also discussed in closer detail by Carnap in his Abriss der Logistik of 1929. In Part II of the book-titled "Applied Logistic"-, Carnap gives a type-theoretic formalization of several axiomatic theories, including different versions of basic arithmetic, set theory, projective geometry, and topology (based on Hausdorff's neighborhood axioms). In light of these theories, Carnap reiterates the point that an axiom system does not only give implicit definitions of the primitive terms occurring in the axioms, but also an explicit definition of a higher-order concept, the Explizitbegriff of 
an axiom system. Put in modern and purely extensional terms, such an explicit concept is understood here as the class of models of a given theory. Thus, the explicit concept defined by the axioms of basic arithmetic is simply the class of all progressions (or number systems), the concept defined by the Hausdorff axioms is the class of all neighborhood spaces, etc.

Carnap's view that axioms define classes of mathematical systems also forms a central tenet of the structuralist conception in contemporary philosophy of mathematics. The main idea here is that an effective way to understand and acquire knowledge of a mathematical structure is by means of a direct description of it. This is achieved by providing an axiomatization of the mathematical theory, which determines-when successful-the class of all its models. The view that mathematics consists in the study of mathematical structures and the structural properties of their objects is then a fundamental motivation for this understanding of structural definitions:

The structuralist orientation of modern mathematics naturally leads to the use of axiomatization. To understand a kind of structure, for instance the structure of a group, is to have an overview over all its instantiations. In an axiomatic system, this is accomplished by capturing all those structures as the models of the system. [...] this method is the natural one for a structuralist. The axioms determine a class of structures as models of the axiom system. By deriving theorems from the axioms a mathematician can study those structures. (Hintikka 2011, p. 70. Our emphasis.)

It is worth mentioning that this conception of structural definitions is particularly suitable for non-eliminative versions of mathematical structuralism, specifically for ante rem structuralism. ${ }^{33}$ Non-eliminative structuralism is the position which considers structures as existing entities in an abstract realm. In particular, according to ante rem structuralism, abstract structures and positions should be conceived as bona fide objects which exist independently of the particular systems of objects which constitutes their concrete instantiations. For the ante rem structuralist, it is then essential that one can communicate and refer to pure structures and positions, independently of their concrete instantiations. Now, a successful structural definition does not define a concrete mathematical system, but the class of the models of the axiomatic theory. Accordingly, in virtue of this model of structural definitions, it is possible to describe pure mathematical structures, even if no particular instance is displayed or known. In the words of Shapiro:

Structures successfully described by implicit definitions are naturally construed as ante rem (if they exist at all, of course). [...] In an implicit definition, asking about Julius Caesar is similar to the aforementioned listener who is wondering about the name of the center fielder's mother. The speaker was describing a structure, not a system of particular people. The mathematics book is not describing a system of sets or Platonic objects or people. It

33 See, for example, Shapiro (1997) and Parsons (1990). 
describes a structure or a class of structures. (Shapiro 1997, pp. 130-132. Our emphasis.)

There is an important connection between the present view of structural definitions and the function of axiomatization in mathematical practice. When axiom systems are considered from a semantic point of view, by focusing on their meaning, one can distinguish between descriptive and prescriptive axiomatizations. ${ }^{34}$ According to the former, the function of an axiomatization is to describe a given domain. According to the latter, the function of an axiom system consists in turn in prescribing or establishing conditions for certain domains, i.e., in determining and defining what the axiomatic theory is about. ${ }^{35}$ The conception of structural definitions as definitions of classes of mathematical structures is then intimately tied to a specific way in which axioms are used in mathematical practice. More specifically, when axioms are considered in their semantic prescriptive dimension, they do not strictly define the meaning of its primitive terms, but the class of models of the axiomatic theory:

If the meanings of the primitives of an axiomatic system depend entirely on the relations that are expressed by the axioms, then the axioms are considered to be prescriptive (or normative), i. e., they determine the subject matter under consideration, and one also speaks of them as 'implicit definitions'. [...] Considered in this way, a system of axioms generally cannot determine a single interpretation for its terms, but only the structure of the relations that hold between them; understood in this way, axiom systems are also called relational, structural, algebraic, or abstract. In other words, such a system of axioms does not define one single model, but a class of models. (Schlimm 2013, pp. 50-51)

Finally, axiom systems can also be considered from a syntactic perspective, by focusing on the deductive role of axioms as the starting points for deducing theorems. According to their syntactic role, axioms are viewed as sentences which determine the propositions that pertain to a theory, i.e., all those sentences that can be deductively obtained from them. Prima facie, the syntactic dimension of axioms has then no bearing on any particular understanding of structural definitions. We will contest this last claim in the following section, by assessing the relevance of different views of meaning in the formal account of structural definitions.

\section{Assessment}

As the last section made clear, structural definitions play an important role in the history of mathematics as well as in analytic philosophy. However, it was also shown that the notion is used only informally in these debates. This is particularly true for recent work in the philosophy of language and mathematics, for instance, on

\footnotetext{
34 This distinction has been recently investigated in Schlimm (2013). A related distinction between assertory and algebraic axiom systems has been defended by Shapiro (2005).

35 See Schlimm (2013, p. 49).
} 
abstraction principles or structuralism. Perhaps more surprisingly, the two notions also lack a rigorous logical explication in modern axiomatic mathematics in the wake of Dedekind's and Hilbert's work.

We saw in the previous section that one can distinguish between two ways of understanding structural definitions, namely (1) as specifications of the meaning of primitive terms of a theory and (2) as specifications of higher-order mathematical concepts or structures (e.g. the concepts of Euclidean space, number progressions, abstract groups, etc.). Given these two types of structural definitions, how can we think of them semantically? In the following, we will provide a more systematic discussion of the semantic understanding of structural definitions, first in terms of model theory and second in terms of an inferential semantics.

\subsection{Structural Definitions and Model Theory}

Can one provide a model-theoretic explication of structural definitions? In contrast to the well-known semantics of implicit definitions, there is no straightforward model-theoretical account of structural definitions. More specifically, it is not prima facie clear how by the stipulation of the consistency of a theory, one also determines the meaning of its primitives terms. Moreover, recall from Sect. 2 that in the case of implicit definitions (in the strict sense), the central model-theoretic condition expresses a uniqueness condition for the interpretation of the defined symbol. This kind of uniqueness of reference is, as we saw, deliberately ruled out in the informal discussions of structural definitions in the work of Hilbert and others, at least if understood as definitions of the primitive terms of a theory.

This lack of a clear semantic specification of structural definitions through axiom systems has led logicians and model theorists to question the validity of this form of definition. Consider, for instance, Hodges' insightful remarks on the historical development of the notion:

Unfortunately there used to be a very confused theory about model-theoretic axioms, that also went under the name of implicit definition. By the end of the nineteenth century, mathematical geometry had generally ceased to be a study of space, and it had become the study of classes of structures which satisfy certain 'geometric' axioms. Geometric terms like 'point', 'line' and 'between' survived, but only as the primitive symbols in axioms; they no longer had any meaning associated with them. (...)

Problems arose because of the way that Hilbert and others described what they were doing. (...) they went on to say that the axioms together form an implicit definition of the concepts plus, minus and 0 together, and that this definition is only partial but it says about these concepts precisely as much as we need to know.

One wonders how it could happen that for fifty years nobody challenged this nonsense. (...) Frege's demolition of the implicit definition doctrine was masterly, but it came too late to save Hilbert from saying, at the beginning of his 
Grundlagen der Geometrie, that his axioms give "the exact and mathematically adequate description" of the relations 'lie', 'between' and 'congruent'. Fortunately, Hilbert's mathematics speaks for itself, and one can simply bypass these philosophical faux pas. (Hodges 2001/2013, §2)

According to Hodges, the "confusion" about the concept of implicit definitions in modern axiomatics really concerns structural definitions in our first sense. This notion simply does not have a precise sense from a model-theoretic point of view. Thus, whereas one can view an axiom system as a definition of a higher-order concept along Fregean lines, it is not possible to think of it as specifying the meaning of the non-logical primitives.

The standard model-theoretic treatment of the Fregean understanding of axiom systems is based on the definability of model classes. The notion was first introduced by Tarski in the 1950 s and is today usually specified as follows: ${ }^{36}$

Definition 4 (Definable classes of structures) Let $T$ be any theory expressed in a formal language $\mathcal{L}$. Let $K$ be a class of $\mathcal{L}$-structures. Then $T$ defines (or axiomatizes) $K$ if $K$ is the class of all $\mathcal{L}$-structures which are models of $T$, that is, if

$$
K=\operatorname{Mod}(T)=\{M \mid M \vDash T\}
$$

A structural definition in the second sense is naturally framed in model theory as the definition of a class of models or structures satisfying the axioms of the theory in question. ${ }^{37}$ Consider again the example of the theory of analysis presented in Sect. 2. What this axiomatic theory defines is the class of its models, that is, the class of all complete ordered fields satisfying the above mentioned condition.

Notice that this model-theoretic account of structural definitions can be traced back to early contributions on the axiomatic method. Hodges, in his discussion of the history of model theory, holds that "[i]t was Hilbert and his school who first exploited axioms, higher-order as well as first-order, as a means of defining classes of structures." (Hodges 2001, p. 69) We saw in Sect. 3.2 that Hilbert's own methodological remarks on this topic in Grundlagen and in related writings remain rather vague. In contrast, Carnap, in his work on axiomatics from the 1920s, effectively identifies the "explicit concept" defined by an axiom system with its class of models.

What about structural definitions in the first sense, as specifications of the meaning of a theory's primitive terms? We saw in Sect. 3.1 that several authors explicitly refer to the notion of meaning in this context without given a further clarification what meaning is supposed to be. Does this notion of structural definitions, as Hodges suggests, therefore simply rest on a confusion with the notion of definable model classes or is there a way of making sense of it model-theoretically? Contra Hodges, we believe that there are in principle two ways to vindicate the notion and

\footnotetext{
36 See Hodges (1993, p. 30).

37 Notice that the notion of model classes is left informal here. See Hodges (1993) for a similar treatment. Many examples of axiomatizable classes of models are, strictly speaking, proper classes and not sets and should thus be studied in a formal class theory such as NBG. We would like to thank an anonymous reviewer for emphasizing this point to us.
} 
to provide a model-theoretic analysis of it. Let us briefly outline each of them in turn.

First, one might argue that structural definitions present special cases of implicit definitions in the strict sense (as outlined in Sect. 2). More specifically, one could understand a structural definition of several primitive terms by an axiomatic theory as a set of implicit definitions (in the strict sense), where each definition in the set specifies the meaning of one primitive term relative to stipulated meanings of the other terms. More formally, given a theory $T$ expressed in a formal language $\mathcal{L}$ with signature $\left\{\beta_{1}, \ldots, \beta_{m}\right\}$, the structural definition of the primitives by $T$ (in symbols $\left.S D\left(\beta_{1}, \ldots, \beta_{m} ; T\right)\right)$ can be schematically presented as follows:

$$
S D\left(\beta_{1}, \ldots, \beta_{m}, T\right)=\left\{\begin{array}{l}
I D_{1}\left(\beta_{1}, T, \mathcal{L} /\left\{\beta_{1}\right\}\right) \\
I D_{2}\left(\beta_{2}, T, \mathcal{L} /\left\{\beta_{2}\right\}\right) \\
\vdots \\
I D_{m}\left(\beta_{m}, T, \mathcal{L} /\left\{\beta_{m}\right\}\right)
\end{array}\right.
$$

where each $I D_{i}$ presents the implicit definition (in the strict sense) of a given primitive term $\beta_{i}$ by the theory $T$ in terms of the remaining signature $\mathcal{L} /\left\{\beta_{i}\right\}$.

Notice that in this interpretation, structural definitions turn out to be proper definitions in the sense that a certain semantic uniqueness condition is met. The reference of any primitive term is uniquely determined here relative to a given interpretation of the other primitive terms. At the same time, the definition is clearly contextual or holistic in the intended sense: the interpretation of each primitive constant can be specified only relative to the interpretation of the other terms and vice versa.

The second approach to a semantic understanding of structural definitions is based on an intensional interpretation of the primitive vocabulary. The key idea here is to take seriously remarks by philosophers such as Schlick or Boghossian to the effect that theories specify the meaning of its basic terms. If this talk about the meaning or sense of primitive terms is taken literally, then one should not think about structural definitions in terms of a referential semantics. The meaning of mathematical terms should not be understood referentially, but rather intensionally.

The standard intensional account of properties and relations goes back to Carnap's Meaning and Necessity (1947) and to Lewis' (1986) possible-worlds approach to properties. Applied to the context of mathematics, a natural way to think of the intension of a given primitive symbol is as a function from the models of a given mathematical theory to sets of tuples of elements in these systems that can be used for the interpretation of the symbol in question. Thus, according to this account, the primitive terms of a theory do not refer to relations or functions understood extensionally as sets in a given model domain, but rather as functions from models to local extensions. 
Following a recent proposal made in Korbmacher and Schiemer (2018), one can capture the mathematical properties or "concepts" (in Hilbert's sense) expressed by the primitive terms of a theory more formally in the following way: ${ }^{38}$

Definition 5 (Mathematical Properties) Let $\mathrm{P}$ be a property of elements of systems of a given mathematical signature $\mathcal{L}$. The local extension of $\mathrm{P}$ in a system $\mathcal{S}$ is the class $\epsilon_{\mathcal{S}} \mathrm{P}=\left\{x \in \mathcal{D}^{\mathcal{S}} \mid \mathrm{P}(x)\right\}$ of all and only those things in the domain of $\mathcal{S}$ that have the property. The intension of property $\mathrm{P}$ is the function $\imath \mathrm{P}: \mathcal{S} \mapsto \epsilon_{\mathcal{S}} \mathrm{P}$ that assigns to every system $\mathcal{S}$ of signature $\mathcal{L}$ the local extension $\epsilon_{\mathcal{S}} \mathrm{P}$ of $\mathrm{P}$ in system $\mathcal{S}$ (compare Korbmacher and Schiemer 2018, p. 300).

Given this account of mathematical properties and relations, we can say that a theory $T$ provides a structural definition of its primitive terms in the sense that it specifies their respective intensions. Thus, for instance, the axiom system of Peano arithmetic defines the meaning of the primitive terms 'natural number', 'zero', and 'successor' in the following sense: it (uniquely) determines three functions from PAmodels to the local extensions of these terms in each model. Put more schematically, the meaning of the primitive terms $\left\{\beta_{1}, \ldots, \beta_{n}\right\}$ (i.e. in language $\mathcal{L}$ ) defined by a theory $T$ (as a set of sentences in $\mathcal{L}$ ) is simply a sequence of such functions $\left\{\Phi_{\beta_{1}}, \ldots, \Phi_{\beta_{n}}\right\}$ with the model class $\operatorname{Mod}(T)$ as its domain.

Notice that in this account of structural definitions, the semantic uniqueness condition constitutive for proper definitions is met. However, in the present context, this does not concern the uniqueness of reference relative to a given model. Rather, it concerns the fact that an axiom system identifies a single property or relation as the meaning (or proper interpretation) of each primitive term. These properties and relations are understood as intensional entities however, that is, as functions from models to local extensions.

\subsection{Structural Definitions and Inferentialism}

There is a second possibility of understanding structural definitions, which is motivated by a different account of the way in which linguistic expressions acquire their meaning. This alternative understanding of structural definitions is also intimately related to two different approaches in modern axiomatics. As is well known, one can identify two main traditions within formal axiomatics, both from historical and systematic perspectives. The first considers axiom systems primarily from a semantic standpoint, as a way to define the subject-matter of a theory. This perspective focuses on the referents of the axioms, that is, on providing a clear understanding of what precisely a particular axiom system refers to. On the contrary, the second tradition grants a privilege to the proof-theoretical function or role of axioms.

\footnotetext{
38 In order to keep the discussion simple, we restrict our attention here to properties or unary relations. Similar definitions can be given for relations and functions of any arity.
} 
Accordingly, axiom systems are mainly conceived as tools or devices to prove theorems. $^{39}$

These two perspectives on the roles of axioms in modern axiomatics can be illustrated by considering a simple mathematical example. In geometry, an affine plane $\mathcal{A}$ is a system of points and lines together with an incidence relation between points and lines such that the existence of non-intersecting "parallel" lines is allowed. More formally, if $\mathbf{P}$ and $\mathbf{L}$ are two one-place predicates which stand respectively for "points" and "lines", and I is a two-place predicate which designates the relation of points "lying on" a line, then an affine plane is an incidence structure $\langle\mathbf{P}, \mathbf{L}, \mathbf{I}\rangle$ which satisfies the following axioms:

(AP.1) Given two distinct points $P$ and $Q$, there exists a unique line $l$ such that $P$ lies on $l$ and $Q$ lies on $l$.

(AP.2) Given any line $l$ and any point $P$ not on $l$ there exists one and only one line $m$ such that $Q$ is on $m$, and $l$ and $m$ have no common point.

(AP.3) There exist three non-collinear points. ${ }^{40}$

From a semantic perspective, one shall focus then on the different domains or systems of objects which satisfy the conditions prescribed by the axioms, that is, on the models of the axioms. This includes, for instance, the usual Euclidean plane, the Moulton plane ${ }^{41}$, as well as finite models consisting only of four points and six lines. ${ }^{42}$ On the contrary, when the syntactic dimension of axioms as the starting points of mathematical deductions is the central aspect, one might investigate which immediate consequences can be obtained by means of these conditions. For example, one can prove that parallelism is an equivalence relation by using axiom (AP.2) and the definition of parallelism: a line $l$ is parallel to a line $m$ (denoted $l \| m$ ) if either they are equal $(l=m)$ or the two lines have no points in common.

\section{Theorem 1 "Parallel" is an equivalence relation.}

Proof By definition "parallel" is clearly reflexive and symmetric. To prove that this relation is also transitive, let us assume that $l_{1}, l_{2}, l_{3}$ are three distinct lines and that $l_{1} \| l_{2}$ and $l_{2} \| l_{3}$. If $l_{1} H l_{3}$, then they meet at a common point $P$. But this implies

\footnotetext{
${ }^{39}$ For a detailed analysis of these two traditions in modern axiomatics, and its historical and conceptual connections with late nineteenth-century mathematical practice, see Schlimm (2013) and Blanchette (2017).

40 These axioms are a slight modification of the axioms given in Artin (1957, pp. 52-53). There are several ways to axiomatize affine planes, but the present one is the most convenient for our purposes.

${ }^{41}$ The Moulton plane is an affine plane in which Desargues' theorem does not hold. For a historical account of this model, see Arana and Mancosu (2012).

${ }^{42}$ In fact, the smallest affine plane has exactly four points and six lines, and can be described synthetically as follows: $\mathcal{P}=\{A, B, C, D\}$ and $\mathcal{L}=\{\{A B\},\{A C\},\{A D\},\{B C\},\{B D\},\{C D\}\}$. For details on finite models of affine planes, see Bennett (1995).
} 
that $l_{1}$ and $l_{3}$ are two distinct lines through a point $P$ parallel to $l_{2}$, which is impossible by axiom AP2. Then, $l_{1} \| l_{3}$.

The tradition that conceives axioms mainly as inference tools in demonstrations is known as deductivism or if-thenism, that is, the view that mathematics is strictly speaking the study of the logical consequences that can be obtained from a set of given formal or uninterpreted axioms. ${ }^{43}$ Historically, Moritz Pasch's axiomatic investigations into the foundations of projective geometry, in his book Vorlesungen über neuere Geometrie (1926), are usually credited as the earliest and clearest example of this conception within modern axiomatics. In an often quoted passage, Pasch makes the following observation regarding the nature of proofs in axiomatic geometry:

If geometry is to be truly deductive, the process of inference must be independent in all its parts from the meaning of the geometrical concepts, just as it must be independent of the diagrams; only the relations specified in the propositions and definitions may legitimately be taken into account. During the deduction it is useful and legitimate, but in no way necessary to think of the meanings of the terms; in fact, if it is necessary to do so, the inadequacy of the proof is made manifest. (Pasch 1926, p. 91)

Pasch's notable insight consisted in noticing that, when the focus is set on the inferential role of axioms, their meaning is completely irrelevant for the process of deduction. More precisely, rigor in demonstrations can only be achieved if mathematical inferences are carried out independently of any particular sense and reference that might be attached to the primitives concepts occurring in the axioms. Now, given this way of understanding the function of axioms in mathematics, a natural way to proceed is not only to claim that the meaning is not required for deductions, but also to take a step forward and claim that the axioms, as kinds of rules of inference, constitute the meaning of the primitives. In other words, one could claim that the meaning of (geometrical) primitives is determined precisely by the specific inferential patterns prescribed in the axioms, when they are considered from a strictly syntactic viewpoint.

This view is, in fact, widespread in relation to the understanding of logical constants, specifically in the inferentialist conception of the meaning of a logical constants. According to logical inferentialism, the meaning of logical constant can be equated with the usual rules of inference that govern their correct use. Thus, for instance, the meaning of 'and' is determined by its introduction and elimination rules, and to understand the meaning of this logical expression is to apply correctly these rules of inference. The inferential account of the meaning of logical constants has its roots in G. Gentzen's (1935) famous suggestion that the

\footnotetext{
43 See Putnam (1967) and Musgrave (1977) for detailed discussions of the if-thenist conception of mathematics. Compare, in particular, Musgrave's analysis of the critical impact of Gödel's first incompleteness theorem on if-thenism as well as of possible attempts to vindicate the position.
} 
introduction rules, in his systems of natural deduction, could be taken as "definitions" of the respective logical operator:

To every logical symbol $\&, \vee, \forall, \exists, \rightarrow, \neg$, belongs precisely one inference figure which 'introduces' the symbol—as the terminal symbol of a formula—and one which 'eliminates' it. [...] The introductions represent, as it were, the 'definitions' of the symbols concerned, and the eliminations are no more, in the final analysis, than the consequences of these definitions. (Gentzen 1935, p. 80)

There is, an important observation to be made here regarding our discussion of structural definitions. The semantic tradition in modern axiomatics is clearly connected with a model-theoretic reading of structural definitions, in particular, with the second understanding of structural definitions as definitions of higher-order concepts or classes of structures. On the contrary, the syntactic or "proof-theoretic" perspective is more related to the first conception of structural definitions, that is, as specifications of the meaning of the primitive terms of a (mathematical) theory. But an essential point is that this understanding of structural definitions rests upon an inferential account of semantics, or more broadly, on a "use-theoretic" view of meaning, according to which the meaning of linguistic expressions is essentially determined by the basic regularities and rules of its use. In a nutshell, while a "model-theoretic" or "truth-theoretic" stance on meaning naturally leads to the second conception of structural definitions, a "use-theoretic" view of meaning, and in particular an inferential role semantics, leans towards the first conception. ${ }^{44}$

It should be mentioned that the proposal of explaining structural definitions in the first sense, not in a model-theoretic fashion, but in terms of an inferential view of meaning, has been already suggested in the recent philosophical literature. Particularly, Horwich $(1997,1998)$ has argued that many important problems faced by the "standard" or truth-theoretic model of implicit definitions (Cf. Sect. 3.1) can be surmounted by adopting a "use-theoretic" conception of implicit definitions. According to the latter model, the specification of the meaning of a term by means of the assertion of a sentence (or sentences) containing it amounts to establishing a set of regularities for its correct use. More precisely, if the set of sentences comprising the implicit definition is a given (scientific or mathematical) theory, then the meaning of the defined term consists precisely in its theoretical role in such a theory. Then, the central contention of this "use-theoretic" model is that in an implicit or contextual definition a new term acquires its meaning just by regarding the sentence (or sentences), in which it occurs, as true. In other words, this view denies that the statements which comprise the implicit definition have to be true in order to be successful in providing a meaning for the definiendum. ${ }^{45}$

\footnotetext{
44 For a very informative account of the "use-theoretic" approach to semantics, in particular, to inferential role semantics, see Murzi and Steinberger (2017).

45 Cf. Ebert (2016), p. 140. Ebert (2016) has elaborated on Horwich suggestion of applying a "use-theoretic" view of meaning for explaining the concept of implicit definition, and has provided a general framework for the notions, distinguishing several semantic and epistemic dimensions.
} 
Now, a striking trait in the former attempts to provide an account of implicit definitions on the basis of an inferential view of meaning is that the resulting conception is not related primarily to formal languages. Thus, it remains to be determined whether such notions can be adequately characterized in more formal terms, in relation to specific formal languages. Moreover, logical inferentialism has proved to be an appealing view regarding the nature of the meaning of logical constants. But what about the application of this inferentialist point of view to the case of mathematical theories?

While we cannot present a worked-out theory of an inferential semantics for axiomatic theories in the present paper, it should be noted here that such an approach has been suggested in the literature on inferentialism. In particular, Peregrin (2014) contains a discussion of the inferential role of mathematical axioms in the above sense. Understood in this way, they can also serve as specifying the meaning of the primitive vocabulary described in the axioms. For instance, given the example of Peano arithmetic, Peregrin argues that one could "see its axioms as a complex inferential pattern characterizing its basic vocabulary: 0, S, +, •" (Peregrin 2014, p. 54). A central difference to logical inferentialism is that the meaning of terms is specified here not in terms of a rule-based calculus, but rather in terms of several axiomatic conditions. Moreover, Peregrin's proposal also differs from more familiar rule-based versions of inferentialism in the kind of holism implied by it. Instead of specifying a single operator in terms of its corresponding pair of introduction and elimination rules, an axiom system is supposed to give a holistic specification of the meaning of all of its primitive terms.

\section{Conclusion}

The central aim in this paper was to give a historically informed answer to the question "What are implicit (or structural) definitions?". More specifically, we analyzed how the view that axiom systems present definitions was understood in mathematics as well as in modern analytic philosophy. In the context of modern axiomatics, we saw that it makes sense to distinguish between different types of definitions through an axiom system. These are, on the one hand, implicit definitions in the strict sense, that is, definitions of newly introduced terms based on a given interpretation of the primitive vocabulary in some base language. On the other hand, we take structural definitions to be definitions through an axiom system that are independent of any prior semantic stipulations.

The history of formal axiomatics surrounding Hilbert's work shows that one should further distinguish between two ways of understanding structural definitions, depending on what the definiens of such definitions is taken to be. According to the first approach, axiom systems provide a holistic definition of the meanings of its primitive terms. In the second approach, they are viewed as definitions of higherorder mathematical concepts or structures. For instance, in Dedekind's foundational work on arithmetic, the system of axiomatic conditions is explicitly described by 
him as a definition of a higher-order concept, namely the concept of a simply infinite (number) system.

Interestingly, both ways of thinking about structural definitions in modern axiomatics can also be identified in modern debates in analytic philosophy. Specifically, we saw that the notion of implicit definition (construed as a form of structural definitions in the first sense) plays a crucial role in Boghossian's and Horwich's work on analyticity. A similar notion is used again in neologicism, in particular in the discussion of the status of abstraction principles such as Hume's principle. Analogously, the notion of structural definitions in the second sense is employed in modern contributions to the philosophy of mathematics, most explicitly in Shapiro's discussion of non-eliminative structuralism.

Based on the survey of the different uses of the notions in modern mathematics and analytic philosophy, we gave a more systematic assessment of the underlying semantics of the two ways of understanding the definiens of such definitions. Whereas the model-theoretic reconstruction of structural definitions in the second sense as definitions of model classes is straightforward, the semantic interpretation of structural definitions in the first sense is significantly less clear. In the paper, we outlined two possible ways of understanding such definitions semantically. According to the first, model-theoretic account, the meaning of a primitive term defined through an axiom system is understood not referentially, but rather intensionally as a function from models to local extensions. According to the second, inferentialist account, the meaning of the primitive terms is not specified model-theoretically at all. Instead, their meaning is determined by their inferential role in deducing theoretical consequences from the axiom system in question.

Both the intensional and the inferentialist accounts of axiomatic definitionsunderstood as definitions of the meaning of the primitive terms of a theory-have only been sketched briefly here. A more thorough analysis of the different semantics of structural definitions resulting from them has to be given in a different paper.

Acknowledgements Open access funding provided by University of Vienna. This project has received funding from the European Research Council (ERC) under the European Union's Horizon 2020 research and innovation programme (Grant Agreement No. 715222). We would like to thank Francesca Biagioli, Paola Cantù, Vincenzo De Risi, Günther Eder, Marco Giovanelli, Ansten Klev, Dirk Schlimm, John Wigglesworth, and two anonymous referees for their insighful comments and suggestions on previous versions of this paper. Lastly, the first author thanks the Austrian Academy of Sciences (ÖAW), for its support during work on this project.

Open Access This article is distributed under the terms of the Creative Commons Attribution 4.0 International License (http://creativecommons.org/licenses/by/4.0/), which permits unrestricted use, distribution, and reproduction in any medium, provided you give appropriate credit to the original author(s) and the source, provide a link to the Creative Commons license, and indicate if changes were made.

\section{References}

Antonelli, A. (1998). Definitions. In E. Craig (Ed.), Routledge encyclopedia, Logic \& mathematics (pp. 150-154). London: Routledge.

Arana, A., \& Mancosu, P. (2012). On the relationship between plane and solid geometry. The Review of Symbolic Logic, 5, 294-353. 
Artin, E. (1957). Geometric algebra. New York: Interscience Publishers.

Awodey, S., \& Reck, E. (2002). Completeness and categoricity. Part I: Nineteenth-century axiomatics to twentieth-century metalogic. History and Philosophy of Logic, 23, 1-30.

Belnap, N. (1993). On rigorous definitions. Philosophical Studies, 72, 115-146.

Ben-Menahem, Y. (2006). Conventionalism. New York: Cambridge University Press.

Bennett, M. K. (1995). Affine and projective geometry. New York: Interscienec Publications.

Blanchette, P. (2017). Models in geometry and logic: 1870-1920. In N. S. Sober (Ed.), Logic, methodology and philosophy of science-Proceedings of the 15th international congress (pp. 41-61). London: College Publications.

Boghossian, P. (1996). Analyticity reconsidered. Nô̂s, 30, 360-391.

Boghossian, P. (1997). Analyticity. In B. Hale \& C. Wright (Eds.), A companion to the philosophy of language (1st ed., pp. 331-368). Oxford: Blackwell.

Burali-Forti, C. (1901). Sur les différentes methods logiques pour la definition du nombre reel. In Congrès International de Philosophie (pp. 289-307). Paris: Colin.

Carnap, R. (1927). Eigentliche und uneigentliche Begriffe. Symposion, 1, 355-374.

Carnap, R. (1929). Abriss der Logistik. Wien: Springer.

Carnap, R. (1947). Meaning and necessity: A study in semantics and modal logic. Chicago: University of Chicago Press.

Coffa, A. (1991). In Linda Wessels (Ed.), The semantic tradition from Kant to Carnap: To the Vienna station. Cambridge: Cambridge University Press.

Dedekind, R. (1888). Was sind und was sollen die Zahlen? Braunschweig: Vieweg. English translation by W. W. Beman, revised by W. Ewald, 1996, pp. 787-833.

Ebert, P. (2016). A framework for implicit definitions and the a priori. In P. Ebert \& M. Rossberg (Eds.), Abstractionism. Essays in philosophy of mathematics (pp. 134-160). Oxford: Oxford University Press.

Ebert, P., \& Shapiro, S. (2009). The good, the bad and the ugly. Synthese, 170, 415-441.

Eder, G. (2016). Frege's 'on the foundations of geometry' and axiomatic metatheory. Mind, 125, 5-40.

Enriques, F. (1907). Prinzipien der Geometrie. In W. Meyer \& H. Mohrmann (Eds.), Enzyklopädie der mathematischen Wissenschaften (Vol. 3.1.1, pp. 6-126). Leipzig: Teubner.

Ewald, W. (1996). From Kant to Hilbert: A source book in mathematics (Vol. 2). Oxford: Clarendon Press.

Frege, G. (1903). Über die Grundlagen der Geometrie, Jahresbericht der Deutschen Mathematiker-Vereinigung, 12, 319-324, 368-375. (Translated by E. H. W. Kluge, Gottlob Frege on the Foundations of Geometry and Formal Theories of Arithmetic (pp. 22-37). New Haven: Yale University Press, 1971).

Frege. G. (1980). Philosophical and mathematical correspondence. Oxford: Basil Blackwell. (Edited by G. Gabriel, H. Hermes, F. Kambartel, Ch. Thiel, A. Veraart).

Freidman, M. (1999). Reconsidering logical positivism. Cambridge: Cambridge University Press.

Gabriel, G. (1978). Implizite Definitionen-Eine Verwechselungsgeschichte. Annals of Science, 35, 419-423.

Gentzen, G. (1935). Untersuchungen über das logische Schliessen. Mathematische Zeitschrift, 39, 176210, 405-431. (Cited from the English translation by M. Szabo, in The Collected Papers of Gerhard Gentzen, Amsterdam: North-Holland, 1969).

Hale, B., \& Wright, C. (2000). Implicit definition and the a priori. In P. Boghossian \& C. Peacocke (Eds.), New essays on the a priori (pp. 286-319). Oxford: Clarendon Press.

Hallett, M. (2010). Frege and Hilbert. In M. Potter \& T. Ricketts (Eds.), The Cambridge companion to Frege (pp. 413-464). New York: Cambridge University Press.

Hallett, M. (2012). More on Frege and Hilbert. In M. Frappier, D. Brown, \& R. DiSalle (Eds.), Analysis and interpretation in the exact sciences (pp. 135-162). New York: Springer.

Hallett, M., \& Majer, U. (Eds.). (2004). David Hilbert's lectures on the foundations of geometry, 18911902. Berlin: Springer.

Hilbert, D. (1899). Grundlagen der Geometrie. In Festschrift zur Feier der Enthüllung des Gauss-Weber Denkmals in Göttingen. Herausgegeben von dem Fest-Comitee. Leipzig: Teubner. Reprinted in Hallett \& Majer 2004 (pp. 426-525).

Hilbert, D. (1900a). Über den Zahlbegriff. Jahresbericht der Deutschen Mathematiker-Vereinigung, 8, $180-184$. 
Hilbert, D. (1900b). Mathematische Probleme. Nachrichten von der königlichen Gesellschaft der Wissenschaft zu Göttingen, mathematisch-physikalische Klasse, 3, 253-296. (English trans. by Mary Winston in Bulletin of the American Mathematical Society, 8, 437-479).

Hilbert, D. (1905a). Logische Principien des mathematischen Denkens; (Vorlesung, SS 1905). Ausgearbeitet von M. Born, Staats-und Universitätsbiblioteck Göttingen, Handschriftenabteilung, Cod. Ms. D. Hilbert 558 a.

Hilbert, D. (1905b). Logische Principien des mathematischen Denkens; (Vorlesung, SS 1905). Ausgearbeitet von E. Hellinger: Georg-August-Universität Göttingen, Mathematisches Institut, Lesesaal.

Hilbert, D. (1971). Foundations of geometry. (La Salle: Open Court. (Translated by L. Unger from the 10th German Edition).

Hilbert, D. (1988). Wissen und mathematisches Denken. (Ausgearbeitet von W. Ackermann. Mohr: Göttingen.)

Hintikka, J. (2011). What is the axiomatic method? Synthese, 183, 69-85.

Hodges, W. (1993). Model theory. Cambridge: Cambridge University Press.

Hodges, W. (2001/2013). Model theory. In E. N. Zalta (Ed.), The Stanford encyclopedia of philosophy (Fall 2018 Edition). https://plato.stanford.edu/archives/fall2018/entries/model-theory/.

Hodges, W. (2008). Tarski's theory of definitions. In D. Patterson (Ed.), New essays on Tarski and philosophy (pp. 94-132). Oxford: Oxford University Press.

Horwich, P. (1997). Implicit definition, analytic truth, and a priori knowledge. Noûs, 31, 423-440.

Horwich, P. (1998). Meaning. Oxford: Oxford University Press.

Klev, A. (2011). Dedekind and Hilbert on the foundations of the deductive sciences. The Review of Symbolic Logic, 4, 645-681.

Klev, A. (2017). Dedekind's logicism. Philosophia Mathematica, 25, 341-368.

Korbmacher, J., \& Schiemer, G. (2018). What are structural properties? Philosophia Mathematica, 26, 295-323.

Lewis, D. (1986). On the plurality of worlds. Oxford: Blackwell.

MacFarlane, J. (2009). Double vision: Two questions about the neo-Fregean program. Synthese, 170, 443-456.

Murzi, J., \& Steinberger, F. (2017). Inferentialism. In B. Hale, C. Wright, \& A. Miller (Eds.), A companion to the philosophy of language (2nd ed., pp. 197-224). Oxford: Wiley-Blackwell.

Musgrave, A. (1977). Logicism revisited. The British Journal for the Philosophy of Science, 28(2), 99-127.

Padoa, A. (1900). Essai d'une théorie algébrique des nombres entiers, précedé d'une introduction logique à une théorie déductive quelconque, Bibliothèque du Congrès international de philosophie, Paris, 1900, vol. 3, Armand Colin, Paris 1902, pp. 309-365. (Translated in part as 'Logical introduction to any deductive theory' in J. van Heijenoort (Ed.), From Frege to Gödel (pp. 118-23) Cambridge, MA: Harvard University Press, 1967).

Parsons, C. (1990). The structuralist view of mathematical objects. Synthese, 84, 303-346.

Pasch, M. (1926). Vorlesungen über neuere Geometrie (2nd ed.). Leipzig: Teubner.

Peregrin, J. (2014). Inferentialism. Why rules matter. Hampshire: Palgrave Macmillan.

Putnam, H. (1967). Mathematics without foundations. The Journal of Philosophy, 64(1), 5-22.

Resnik, M. (1974). The Frege-Hilbert controversy. Philosophy and Phenemenological Research, 34, 386-403.

Schlick, M. (1918). Allgemeine Erkenntnislehre. (Second edition, Berlin, 1925). (Translated from the 2nd edition by A. E. Blumberg, General Theory of Knowledge, Vienna: Springer, 1974).

Schlimm, D. (2013). Axioms in mathematical practice. Philosophia Mathematica, 21, 37-92.

Shapiro, S. (1991). Foundations without foundationalism: A case for second-order logic. New York: Oxford University Press.

Shapiro, S. (1997). Philosophy of mathematics: Structure and ontology. New York: Oxford University Press.

Shapiro, S. (2005). Categories, structures, and the Frege-Hilbert controversy: The status of meta-mathematics. Philosophia Mathematica, 13, 61-77.

Shapiro, S. (unpublished notes). Implicit definition and abstraction.

Sieg, W. (2009). Hilbert's proof theory. In D. Gabbay (Ed.), The handbook of the history of logic (Vol. 5, pp. 321-384)., Logic from Russell to Church Amsterdam: Elsevier.

Sieg, W., \& Morris, R. (2018). Dedekind's structuralism: Creating concepts and deriving theorems. In E. Reck (Ed.), Logic, philosophy of mathematics, and their history: Essays in honor W.W. Tait (pp. 251-301). London: College Publications. 
Sieg, W., \& Schlimm, D. (2014). Dedekind's abstract concepts: Models and mappings. Philosophia Mathematica, 25, 292-317.

Tarski, A. (1994). Introduction to logic and to the methodology of deductive sciences (4th ed.). Oxford/ New York: Oxford University Press.

Publisher's Note Springer Nature remains neutral with regard to jurisdictional claims in published maps and institutional affiliations. 